\title{
eSNR Improvement in Indirect Detection of mid-IR Signals by Wavelength Conversion in SOS Waveguides
}

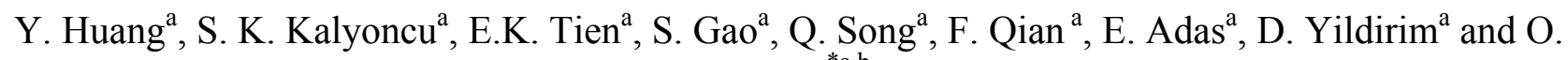 \\ Boyraz ${ }^{* a, b}$ \\ ${ }^{a}$ EECS Department, University of California, Irvine, 92697, USA \\ ${ }^{\mathrm{b}}$ Also founding member of EE Department at Istanbul Sehir University, Istanbul, Turkey \\ Email: oboyraz@uci.edu
}

\begin{abstract}
With a transparency window up to $6 \mu \mathrm{m}$, sapphire can serve as a platform to support silicon photonic integrated circuit in MWIR. Planar waveguide devices based on silicon-on-sapphire (SOS) are emerging as a bridge between MWIR and SWIR through frequency band conversion process. While these devices are widely proposed to amplify MWIR signals and generate MWIR source, it can also be inversely utilized to achieve MWIR light detection. Here MWIR signals are down-converted to telecommunication wavelength $(1.55 \mu \mathrm{m})$ through SOS waveguides and indirectly detected by SWIR detectors. Since detectors at telecommunication wavelengths exhibit superior performances in terms of speed, noise and sensitivity, the indirect detection scheme can be a promising candidate to improve the detection performance. In this report, we analyze performance of the indirect detection of MWIR signals by wavelength conversion in SOS waveguides. Particularly we modeled and compared the noise performance of the indirect detection with direct detection using state-of-the-art MWIR detectors. We show that, in addition to advantages of room temperature and high speed operation, the proposed indirect detection can improve the electrical signal-to-noise ratio up to $50 \mathrm{~dB}, 23 \mathrm{~dB}$ and $4 \mathrm{~dB}$ compared to direct detection by $\mathrm{PbSe}, \mathrm{HgCdTe}$ and $\mathrm{InSb}$ detectors respectively. The improvement is more pronounced in detection of weak MWIR signals.
\end{abstract}

Keywords: MWIR Detection, Silicon waveguide, Noise, wavelength conversion

\section{INTRODUCTION}

Mid-infrared (Mid-IR) spectral range is the window for a wide variety of applications, including free space communication, thermal and biomedical imaging, optical sensing, chemical spectroscopy and military applications, such as missile guidance/countermeasures[1-5]. To facilitate these applications, efficient light detection is a crucial step in mid-IR signal processing. Although commercial detectors are readily available for the whole mid-IR range, their operation condition, noise performance and speed are extremely limited [6]. For example, photovoltaic detectors with narrow bandgap for mid-IR require cooling to suppress dark current and photoconductive detectors are speed-limited by long carrier lifetimes $(\sim 1 \mu \mathrm{s})$. A high-speed and compact solution for low-noise detection is still on-demand. While wavelength down-conversion is widely proposed to amplify mid-IR signals and generate mid-IR source [7-8], it can also be inversely utilized to solve the mid-IR light detection problem [9]. Compared to wavelength conversion in nonlinear crystals, waveguide-based wavelength converters can provide a chip-scale integrated solution with high effective nonlinearity due to its high optical confinement $[8,10]$. However, the mature SOI waveguide technology meets its limitation in mid-IR due to optical loss associated with the buried oxide cladding. Among all proposed alternatives, silicon-on-sapphire (SOS) waveguides are demonstrated to be an excellent candidate, because sapphire has a transparency window up to $6 \mu \mathrm{m}$ and SOS waveguides are compatible with SOI technology [11-12]. Recently, we proposed a frequency band converter based on silicon-on-sapphire (SOS) waveguide capable of converting selected band of mid-IR signals to near-IR $(1.55 \mu \mathrm{m})$ [13]. Based on the converter, here we propose an indirect detection scheme for mid-IR signal by using near-IR detectors. Since detectors at telecommunication wavelengths exhibit superior performances in terms of speed, noise and sensitivity, the indirect detection scheme can be a promising candidate to improve the detection performance. Meanwhile, the four-wave-mixing wavelength conversion process is an instantaneous process and the speed in indirect detection is only limited by the near-IR detectors used after the converter. This enables much faster processing of mid-IR signal than direct detection. Also with the benefit of lower noise in nearIR detectors, the indirect scheme is possible to achieve the same signal-to-noise ratio (SNR) without any cooling.

Infrared Sensors, Devices, and Applications; and Single Photon Imaging II, edited by Paul D. LeVan, Ashok K. Sood, Priyalal S. Wijewarnasuriya, Manijeh Razeghi, Jose Luis Pau Vizcaíno, Rengarajan Sudharsanan, Melville P. Ulmer, Tariq Manzur, Proc. of SPIE Vol. 8155, 81550U - (c) 2011 SPIE · CCC code: 0277-786X/11/\$18 · doi: 10.1117/12.896061 
Here, we analyze limits of indirect detection of mid-IR signals by frequency band conversion in SOS waveguides and investigate signal-to-noise-ratio (SNR) improvement that is attainable with respect to direct detection using state of the art commercial detectors. In particular we provide a comparative study on the noise performance in detection of $4 \mu \mathrm{m}$ mid-IR signals by using PbSe, $\mathrm{HgCdTe}$ and InSb mid-IR detectors, and InGaAs photodetectors following a SOS channel waveguide based wavelength converter. We show that the proposed indirect detection can improve the electrical SNR (eSNR) up to $40 \mathrm{~dB}$ with respect to direct detection, where the improvement is more pronounced in detection of weak incident signals.

\section{DETECTION SETUP}

Figure 1 illustrates the proposed detection scheme. The upper branch illustrates the indirect detection of mid-IR signals up-converted to near-IR wavelengths and detected by telecommunication detectors (InGaAs p-i-n detectors most widely used). Here the wavelength conversion is generated through FWM based modulational instability at SOS waveguides. This process is governed by a slightly modified Nonlinear Schrödinger Equation for ultra-wide frequency band conversion as shown below:

$$
\begin{gathered}
\frac{d A_{p}}{d z}=-\frac{\alpha_{p}}{2} A_{p}+\frac{j 2 \pi n_{2}}{\lambda_{p}}\left[\frac{\left|A_{p}\right|^{2}}{A_{\text {eff }}^{p p}}+\frac{2\left|A_{s}\right|^{2}}{A_{\text {eff }}^{s p}}+\frac{2\left|A_{c}\right|^{2}}{A_{\text {eff }}^{c p}}\right] A_{p}+\frac{j 2 \pi n_{2}}{\lambda_{p} A_{\text {eff }}^{\text {fwm }} A_{p}^{*} A_{s} A_{c} e^{j \Delta k z}} \\
\frac{d A_{s}}{d z}=-\frac{\alpha_{s}}{2} A_{s}+\frac{j 2 \pi n_{2}}{\lambda_{s}}\left[\frac{\left|A_{s}\right|^{2}}{A_{\text {eff }}^{s s}}+\frac{2\left|A_{p}\right|^{2}}{A_{\text {eff }}^{p s}}+\frac{2\left|A_{c}\right|^{2}}{A_{\text {eff }}^{c s}}\right] A_{s}+2 \frac{j 2 \pi n_{2}}{\lambda_{s} A_{\text {eff }}^{\text {fwm }} A_{p} A_{p} A_{c}^{*} e^{-j \Delta k z}} \\
\frac{d A_{c}}{d z}=-\frac{\alpha_{c}}{2} A_{c}+\frac{j 2 \pi n_{2}}{\lambda_{c}}\left[\frac{\left|A_{c}\right|^{2}}{A_{\text {eff }}^{c c}}+\frac{2\left|A_{p}\right|^{2}}{A_{\text {eff }}^{p c}}+\frac{2\left|A_{s}\right|^{2}}{A_{\text {eff }}^{s c}}\right] A_{c}+2 \frac{j 2 \pi n_{2}}{\lambda_{c} A_{\text {eff }}^{\text {fwm }} A_{p} A_{p} A_{s}^{*} e^{-j \Delta k z}}
\end{gathered}
$$

As illustrated previously, dispersion engineering on waveguides can alter the dispersion curve to meet the phasematching condition for different mid-IR signal wavelengths and facilitate frequency band conversion [13-14]. The dispersion of the designed waveguide is calculated with FEM method in COMSOL. A typical dispersion curve is shown in Figure 2. The modal profile demonstrates a good confinement and the blue dispersion curve shows a very flat profile in the mid-IR range at the same time. With this flat dispersion cure, we can achieve mid-IR wavelength conversion with wide bandwidth. For instance, Figure 3(a) shows the conversion efficiency from mid-IR to telecommunication wavelength that can be achieved in different waveguide geometries. Even with only 1D freedom on waveguide width, it is possible to convert mid-IR signal between $2 \mu \mathrm{m}$ and $6 \mu \mathrm{m}$ to near-IR wavelengths of $1.55 \mu \mathrm{m}$, and hence such conversion ability renders itself a suitable platform to generate and detect mid-IR signals for many applications such as free space communication and optical sensing. The dark region in the figure corresponds to the pump wavelength region to enable the conversion from the peaked mid-IR to telecommunication wavelength. Thulium-doped fiber lasers and Chromium-based solid-state lasers can provide adequate pumping in this region [15]. Figure 3(b) shows the conversion efficiency and peak signal wavelength dependence which can be tuned by increasing pump intensity [13].

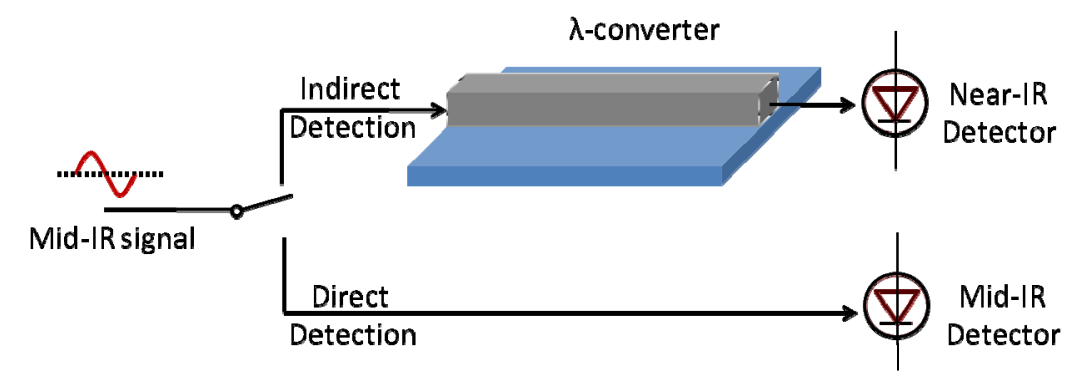

Figure 1. Schematics of direct and indirect mid-IR detection. 


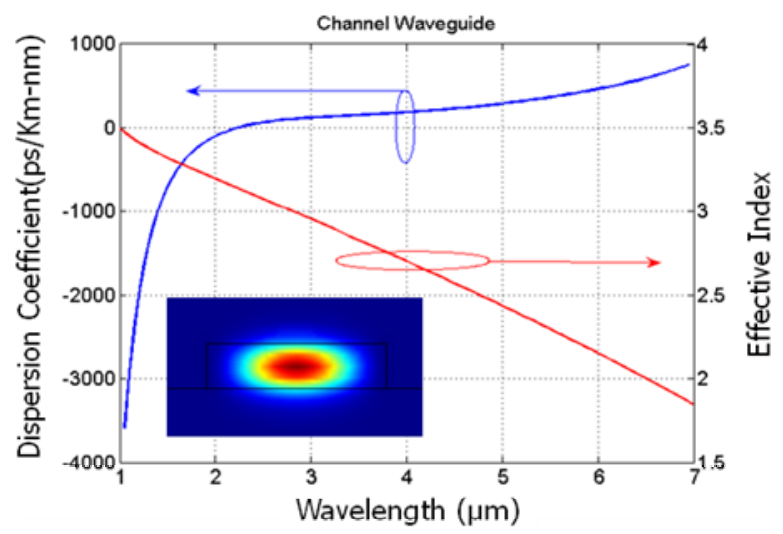

Figure 2. Dispersion of channel waveguide (blue: dispersion coefficient, red: effective index, inset: modal profile)
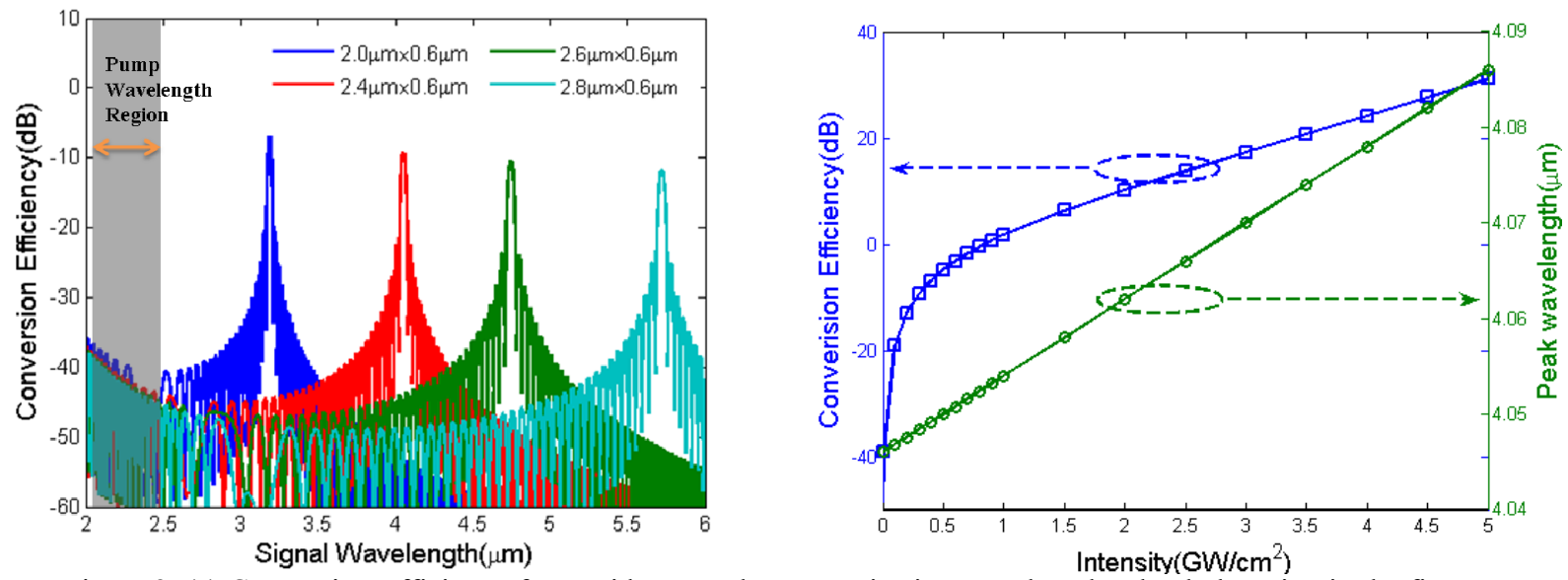

Figure 3. (a) Conversion Efficiency from mid-IR to telecommunication wavelength. The dark region in the figure corresponds to the pump wavelength region to enable the conversion from the peaked mid-IR to telecommunication wavelength. (b) Conversion efficiency (blue) and peak wavelength (green) dependence on pump intensity.

\section{NOISE ANALYSIS}

Noise in indirect detection may originate from wavelength conversion process or the detector itself. Noise associated with wavelength conversion includes quantum noise, Raman induced noise and pump transferred noise. Since the frequency separation is more than three times wider than the Raman shift in silicon the Raman induced noise is negligible. Also, the noise transfer from pump is considered to be detrimental for large signal powers, pump lasers with $\mathrm{RIN}>120 \mathrm{~dB} / \mathrm{Hz}$ and large phase noise [16-17]. Since the noise performance of mid-IR pump lasers is not well characterized we intentionally exclude the noise originating from the pump lasers in our calculations. However, excess quantum noise is always present in the parametric process and will be transferred to InGaAs-based p-i-n detector as quantum fluctuations, $i_{n, q t}^{2}$, described as [13]:

$$
i_{n, q t}^{2}=4 R^{2} G_{i} P_{i n} \frac{h v}{2}\left(2 G_{i}+1\right) B_{e}
$$

Here, $R$ is the responsivity of the detector, $B_{e}$ is the electrical bandwidth, $P_{i n}$ is the incident power and $G_{i}$ is the conversion efficiency numerically calculated by solving nonlinear Schrödinger Equation in SOS waveguide as shown in Figure 2(a). Other detector noise components in the InGaAs detector include thermal noise $i_{n, t h}^{2}$ and shot noise $i_{n, s h}^{2}$ as in Equation(2) and (3), in which $R_{L}$ is load resistance, $F_{n}$ is the circuitry noise figure [18]. 


$$
\begin{aligned}
& i_{n, t h}^{2}=4\left(k_{b} T / R_{L}\right) F_{n} B_{e} \\
& i_{n, s h}^{2}=2 q\left(R P_{i n}+i_{d}\right) B_{e}
\end{aligned}
$$

Lower arm in Figure 1 illustrates the direct detection of mid-IR signals. Commercial mid-IR detectors include photoconductive, photovoltaic and thermal detectors. Thermal detectors are too slow for real-time application, and hence only photoconductive and photovoltaic detectors are analyzed here. Photovoltaic detectors absorb photons with energy beyond and near their bandgap to generate current. Because the energy associated with mid-IR photons corresponds to a narrow bandgap, thermal generation can result in considerable dark current. Low temperature operation is always necessary to suppress dark current and the shot noise associated with it. The noise components in photovoltaic detectors include thermal noise, shot noise and flicker noise. If a low-pass filter is inserted to eliminate flicker noise, the total noise $i_{n, p v}^{2}$ will mainly consist of thermal noise $i_{n, t h}^{2}$ and shot noise $i_{n, s h}^{2}$ as in Equation (2) and (3).

In photoconductive mode, photo-generated carriers change the conductivity of the medium and lead to a change on the voltage across the medium. Due to different detection mechanism, the noise composition in photoconductive detectors is also different from photovoltaic ones. Main noise components in photoconductive detectors include thermal noise, generation-recombination noise (GR-noise), background noise, flicker noise and also the noise transferred from voltage supply. While supply voltage induced noise can be minimized to negligible level by using low noise voltage supplies and flicker noise can also be eliminated by low-pass filters, the total noise $i_{n, p c}^{2}$ mainly includes thermal noise $i_{n, t h}^{2}$, GRnoise $i_{n, g r}^{2}$ and background noise $i_{n, b g}^{2}[19-20]$ :

$$
\begin{aligned}
& i_{n, g r}^{2}=\int_{0}^{B_{e}} \frac{4 q I_{0} \Gamma_{G}}{\left(1+4 \pi^{2} f^{2} \tau^{2}\right)} d f=\frac{2 q I_{0} \Gamma_{G}}{\pi \tau} \tan ^{-1}\left(2 \pi B_{e} \tau\right) \\
& i_{n, b g}^{2}=q^{2} \eta A E_{B B} B_{e}
\end{aligned}
$$

Where $\tau$ is average carrier lifetime, $I_{0}$ is the total generation current, $\Gamma_{G}$ is the current gain, $\eta$ is quantum efficiency, $A$ is active detector area, $E_{B B}=\int_{0}^{\lambda_{c}} \varepsilon_{\lambda} \frac{2 \pi c}{\lambda^{4}}\left[\frac{1}{e^{h_{c} / \lambda k_{b} T}-1}\right] d \lambda$ is the total photon flux density with $\lambda_{c}$ being the cut-off wavelength and $\varepsilon_{\lambda}$ the emissivity for the window material.

\section{RESULTS AND DISCUSSION}

To assess the detection limit and evaluate noise performance we used state-of-the-art commercial mid-IR and near-IR photo-detectors in our calculation. In particular, we use InGaAs PIN detector for near-IR detection in indirect scheme. For direct mid-IR detection, three types of detectors are widely used: InSb photovoltaic, PbSe photoconductive $\mathrm{HgCdTe}$ photoconductive detector. While $\mathrm{PbSe}$ and $\mathrm{HgCdTe}$ detectors can work properly at room temperature, InSb detectors usually require liquid-nitrogen or multi-stage TE cooling. Their main parameters of these used detectors are listed in Table 1, which tracks the up-to-date commercial detectors, e.g. Hamamatsu and Vigo[6,21]. An electrical bandwidth of $0.1 \mathrm{MHz}$ is used to accommodate the relatively slow mid-IR detectors. Figure 4 illustrates the noise at InGaAs detector after the wavelength-converter at room temperature with incident power level of $1 \mu \mathrm{W}$. At low pump intensities, the detection system will be limited by shot noise. As pump intensity increases above $0.4 \mathrm{GW} / \mathrm{cm} 2$, the conversion efficiency increases and the quantum noise becomes dominant. Thermal noise is minor and cooling of near-IR detector cannot further improve the noise performance in indirect detection. 


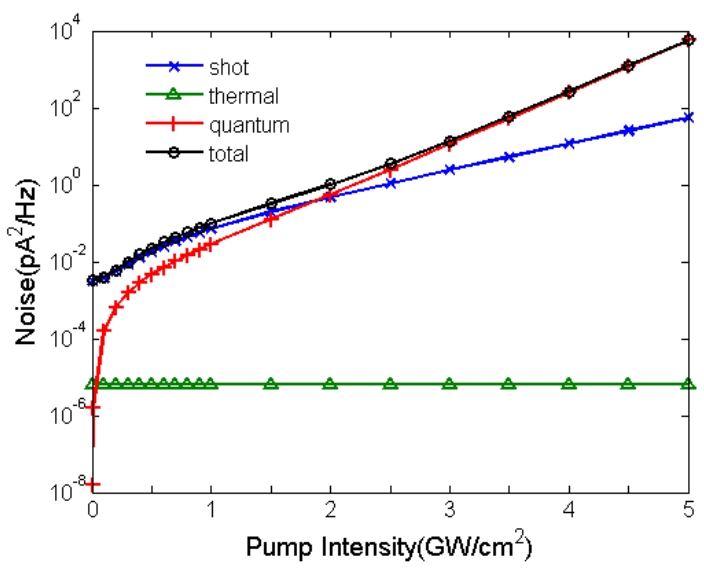

Figure 4. Detection noises at near-IR InGaAs detector in indirect detection

For direct detection at mid-IR, thermal noise dominates in PbSe and $\mathrm{HgCdTe}$ detectors and shot noise is the dominant noise in the liquid nitrogen cooled InSb detectors. We estimate that for the same $1 \mu \mathrm{W}$ input power level at mid-IR the total noise current in PbSe, HgCdTe and InSb detectors to be $0.10 \mathrm{pA} / \sqrt{\mathrm{Hz}}, 4.09 \mathrm{pA} / \sqrt{\mathrm{Hz}}$ and $1.63 \mathrm{pA} / \sqrt{\mathrm{Hz}}$ respectively.

Table I. Detector parameters

\begin{tabular}{lllllll}
\hline & $\mathrm{T}(\mathrm{K})$ & $\lambda_{\text {op }}(\mu \mathrm{m})$ & $\mathrm{R}$ & $\mathrm{R}_{\mathrm{L}}(\Omega)$ & $\mathrm{I}_{\mathrm{d}}$ & $\mathrm{NEP}\left(\mathrm{W} / \mathrm{Hz}^{1 / 2}\right)$ \\
\hline InGaAs & 300 & 1.55 & $0.95[\mathrm{~A} / \mathrm{W}]$ & $8 \mathrm{M}$ & $80 \mathrm{pA}$ & $2 \times 10^{-15}$ \\
$\mathrm{PbSe}$ & 300 & 4.0 & $3 \times 10^{3}[\mathrm{~V} / \mathrm{W}]$ & $0.3 \mathrm{M}$ & $\mathrm{N} / \mathrm{A}$ & $8 \times 10^{-11}$ \\
$\mathrm{HgCdTe}$ & 293 & 4.0 & $3 \times 10^{3}[\mathrm{~V} / \mathrm{W}]$ & $1 \mathrm{~K}$ & $\mathrm{~N} / \mathrm{A}$ & $3.1 \times 10^{-12}$ \\
$\mathrm{InSb}$ & 77 & 4.0 & $2.5[\mathrm{~A} / \mathrm{W}]$ & $1 \mathrm{M}$ & $10 \mu \mathrm{A}$ & $5.5 \times 10^{-13}$ \\
\hline
\end{tabular}

Electrical Signal to Noise Ratio (eSNR) is often used as performance metric. To assess the noise improvement through indirect detection we estimate the eSNR of detectors at three different incident power levels of $0.1 \mu \mathrm{W}, 1 \mu \mathrm{W}$ and $10 \mu \mathrm{W}$ which are measured at the input of mid-IR detectors and at the input of the SOS wavelength converter. The eSNR enhancement is evaluated by comparing the eSNR results of three mid-IR detectors to that of the InGaAs detector as: $e S N R_{\text {near-IR }} / e S N R_{\text {mid-IR }}$, as illustrated in Figure 5(a). At an incident power level of $1 \mu \mathrm{W}$, liquid nitrogen cooled $\mathrm{InSb}$ detectors can achieve about the same eSNR as indirect detection at $1550 \mathrm{~nm}$. However, indirect detection can provide $>10 \mathrm{~dB}$, and $>35 \mathrm{~dB}$ eSNR improvement over direct detection using $\mathrm{HgCdTe}$ and $\mathrm{PbSe}$ detectors, respectively. The eSNR improvement in these detectors is achieved for pump intensities above $0.1 \mathrm{GW} / \mathrm{cm}^{2}$ at which conversion efficiency exceeds $-20 \mathrm{~dB}$. At the pump intensities below $1 \mathrm{GW} / \mathrm{cm}^{2}$ the indirect detection will be limited by thermal and shot noised and we obtain increasing eSNR with increasing conversion efficiency. However, at pump intensities above $1 \mathrm{GW} / \mathrm{cm}^{2}$ the indirect detection will be limited by the quantum noise of the parametric process and the eSNR improvement will saturate at $40 \mathrm{~dB}$ level with respect to PbSe detector for $1 \mu \mathrm{W}$ input power. The advantage of indirect detection will be more obvious for low input power levels. For instance, the SNR improvement with respect to PbSe can go up to $50 \mathrm{~dB}$ for $0.1 \mu \mathrm{W}$ input power levels. Moreover, we even expect that indirect detection can provide $7 \mathrm{~dB}$ eSNR improvement with respect to liquid nitrogen cooled InSb detectors at such low power levels.

The eSNR improvement is wavelength dependent as the conversion efficiency for fixed pump intensity. As Figure 5(b) illustrates, we can attain eSNR improvements within up to $300 \mathrm{~nm}, 200 \mathrm{~nm}$ conversion bandwidths centered at $4 \mu \mathrm{m}$ for input power levels of $0.1 \mu \mathrm{W}$ with respect to $\mathrm{PbSe}$ and InSb detectors, respectively. Although the quantum noise saturates the eSNR improvement at the peak conversion wavelength, eSNR improvement bandwidth increases with increasing pump intensity. This is consistent with the tendency on conversion bandwidth increase with increasing pump intensity [22]. 

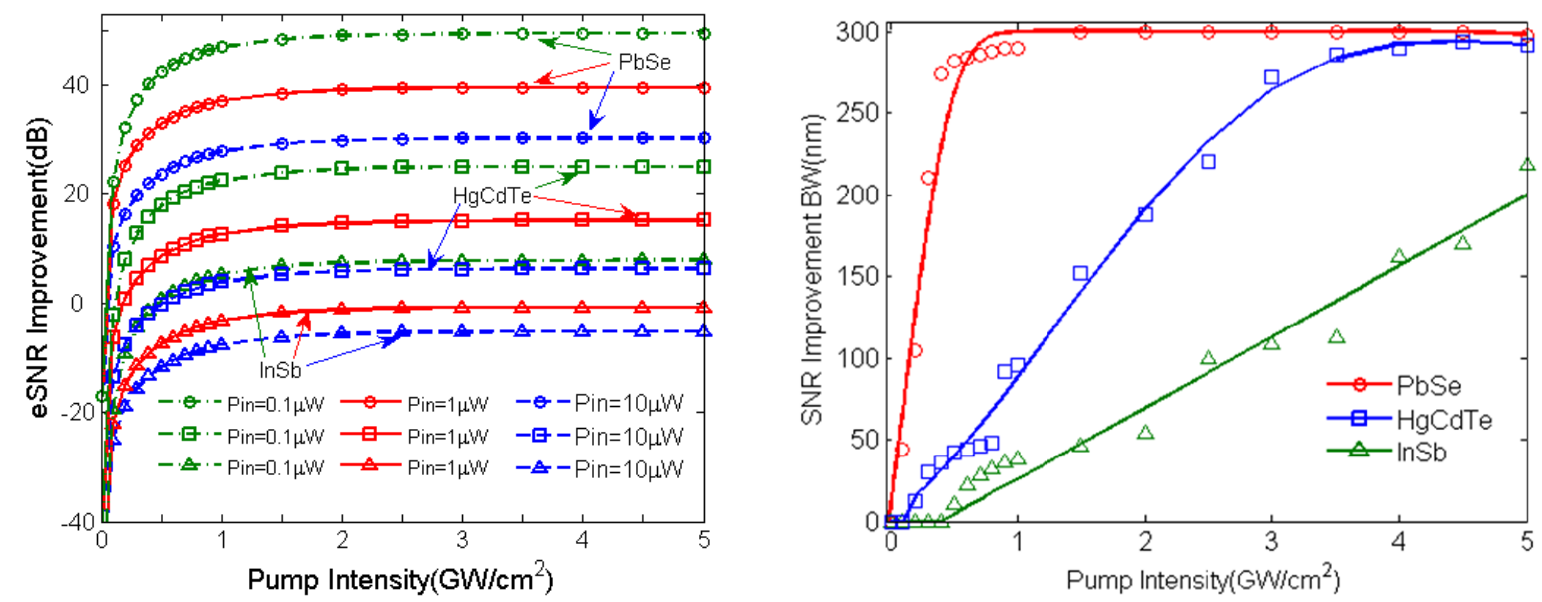

Figure 5. (a) eSNR improvement by using indirect detection with respect to direct detection. $\mathrm{PbSe}$ and $\mathrm{HgCdTe}$ photoconductive detector is set to operate at room temperature and InSb photovoltaic detector is liquid nitrogen cooled to $77 \mathrm{~K}$. (b) Bandwidth on which there is SNR improvement by using indirect detection increase almost linearly with intensity after the threshold and before saturation $(\mathrm{Pin}=0.1 \mu \mathrm{W})$.

\section{SUMMARY}

In summary, Due to high losses, conventional SOI platforms cannot support MWIR photonic IC and planar devices on silicon-on-sapphire (SOS) are emerging as a prospective replacement. Properly-designed SOS waveguides are capable of converting selected MWIR band to SWIR, where the signals are detected with mature telecommunication detectors. We compare the noise performance of this indirect detection with direct detection using MWIR detectors and show that, in addition to room temperature and high speed operation, the proposed indirect detection can improve the electrical signalto-noise ratio up to $50 \mathrm{~dB}, 23 \mathrm{~dB}$ and $4 \mathrm{~dB}$ compared to direct detection using PbSe, HgCdTe and InSb detectors respectively.

Additionally, we compared the noise performance of direct and indirect detection through wavelength conversion of mid-IR signals. At low incident power, indirect detection shows better eSNR performance. At higher incident power, although liquid nitrogen cooled InSb photovoltaic detectors can achieve better SNR, indirect detection is still desirable for high-speed and room-temperature applications. The indirect detection scheme provides a solution for high speed processing of mid-IR signal with good noise performance, which can potentially drive the integration of photonic and electric IC.

\section{ACKNOWLEGEMENT}

This research was supported by DARPA Young Faculty Award (YFA), \#66001-10-1-4036 and EU grant PIRG07-GA-2010-268370.

\section{REFERENCES}

[1] Martini, R.; Bethea, C.; Capasso, F.; Gmachl, C.; Paiella, R.; Whittaker, E.A.; Hwang, H.Y.; Sivco, D.L.; Baillargeon, J.N.; Cho, A.Y.; , "Free-space optical transmission of multimedia satellite data streams using midinfrared quantum cascade lasers," Electronics Letters ,38, 4, pp.181-183, 14 Feb 2002

[2] Yury A. Bakhirkin, Anatoliy A. Kosterev, Chad Roller, Robert F. Curl, and Frank K. Tittel, "Mid-Infrared Quantum Cascade Laser Based Off-Axis Integrated Cavity Output Spectroscopy for Biogenic Nitric Oxide Detection," Appl. Opt. 43, 2257-2266 (2004)

[3] Worrell, C.A.; Giles, I.P.; Adatia, N.A. , "Remote gas sensing with mid-infra-red hollow waveguide," Electronics Letters ,28, 7, pp.615-617, 26 March 1992 
[4] Albert Schliesser, Markus Brehm, Fritz Keilmann, and Daniel van der Weide, "Frequency-comb infrared spectrometer for rapid, remote chemical sensing," Opt. Express 13, 9029-9038 (2005) http://www.opticsinfobase.org/abstract.cfm?URI=oe-13-22-9029

[5] D. H. Titterton, "Development of Infrared Countermeasure Technology and Systems Mid-infrared Semiconductor Optoelectronics", [Springer Series in Optical Sciences], 118, 635-671, DOI: 10.1007/1-84628-209-8_20(2006).

[6] Solid State division, Hamamatsu Photonics K.K., [Characteristics and use of infrared detectors], Tokyo, Japan.

[7] N. K. Hon, K. K. Tsia, D. R. Solli, and B. Jalali, "Periodically poled silicon," Appl. Phys. Lett. 94, 091116 (2009)

[8] X. Liu, R.M. Osgood, Y.A. Vlasov, M. J. GreenWilliam, "Mid-infrared optical parametric amplifier using silicon nanophotonic waveguides", Nat Photon 4, 557-560 (2010).

[9] K. Karstad, A. Stefanov, M. Wegmuller, H. Zbinden, N. Gisin, T. Aellen, M. Beck and J. Faist, Optics and Lasers in Engineering, 43, 537-544.

[10] S.Zlatanovic, J.S. Park, S.Moro, J.M. Chavez Boggio, I.B. Divliansky, N.Alic, S.Mookherjea, S.Radic, "Midinfrared wavelength conversion in silicon waveguides using ultracompact telecom-band-derived pump source", Nat Photon 4, 561-564 (2010).

[11]R. A. Soref, S. J. Emelett, and W. R. Buchwald, "Silicon waveguided components for the long-wave infrared region," Journal of Optics A: Pure and Applied Optics 8, 840-848 (2006).

[12] T. Baehr-Jones, A. Spott, R. Ilic, A. Spott, B. Penkov, W. Asher, and M. Hochberg, "Silicon-on-sapphire integrated waveguides for the mid-infrared," Opt. Express 18, 12127-12135 (2010)

[13]E-K Tien, Y. Huang, S. Gao, Q. Song, F. Qian, S. K. Kalyoncu, and O. Boyraz, "Silicon-on-sapphire integrated waveguides for the mid-infrared," Opt. Express 18, 21981-21989 (2010).

[14] Mohammed N. Islam, and Özdal Boyraz, "Fiber parametric amplifiers for wavelength band conversion" IEEE Journal of Selected Topics in Quantum Electronics, 8, 3,. 527-537(2002).

[15] R. Paschotta, [Encyclopedia of Laser Physics and Technology] (Wiley, Weinheim, Germany,2008)

[16] P. Kylemark, P. O. Hedekvist, H. Sunnerud, M. Karlsson, and P. A. Andrekson, "Noise Characteristics of Fiber Optical Parametric Amplifiers," J. Lightwave Technol. 22, 409- (2004).

[17] S. Moro, A. Peric, N. Alic, B. Stossel, and S. Radic, "Phase noise in fiber-optic parametric amplifiers and converters and its impact on sensing and communication systems," Opt. Express 18, 21449-21460 (2010)

[18] G.P. Agrawal, [Fiber-Optic Communication Systems], Third Edition (Wiley-Interscience 2002).

[19] S. Nudelman, "The Detectivity of Infrared Photodetectors," Appl. Opt. 1, 627-636 (1962).

[20]P. Bhattacharya, [Semiconductor Optoelectronic Devices] (Prentice Hall, Englewood Cliffs, NJ, 1994)

[21] Vigo System, [IR detectors catalogue] (Ozarow Mazowiecki, Poland)

[22]R. H. Stolen and J. E. Bjorkholm, "Parametric amplification and frequency conversion in optical fibers," IEEE J. Quantum Electron. QE-18, 1062-1072 (1982). 